\title{
INTERPOLATION IN AFFINE AND PROJECTIVE SPACE OVER A FINITE FIELD
}

\author{
MICHAEL HELLUS AND ROLF WALDI
}

\begin{abstract}
Let $s(n, q)$ be the smallest number $s$ such that any $n$-fold $\mathbb{F}_{q}$-valued interpolation problem in $\mathbb{P}_{\mathbb{F}_{q}}^{k}$ has a solution of degree $s$, that is: for any pairwise different $\mathbb{F}_{q}$-rational points $P_{1}, \ldots, P_{n}$, there exists a hypersurface $H$ of degree $s$ defined over $\mathbb{F}_{q}$ such that $P_{1}, \ldots, P_{n-1} \in H$ and $P_{n} \notin H$. This function $s(n, q)$ was studied by Kunz and the second author in [8] and completely determined for $q=2$ and $q=3$. For $q \geq 4$, we improve the results from [8].

The affine analogue to $s(n, q)$ is the smallest number $s=s_{a}(n, q)$ such that any $n$-fold $\mathbb{F}_{q}$-valued interpolation problem in $\mathbb{A}^{k}\left(\mathbb{F}_{q}\right), k \in \mathbb{N}_{>0}$ has a polynomial solution of degree $\leq s$. We exactly determine this number.
\end{abstract}

1. Introduction. Let $R=K\left[X_{0}, \ldots, X_{k}\right]$ denote the standard graded polynomial ring in $k+1 \geq 1$ variables over an arbitrary field $K$ and $\mathbb{P}^{k}(K) \subseteq \mathbb{P}_{K}^{k}=\operatorname{Proj} R$ the set of all $K$-rational points.

We start with an arbitrary finite subset $\mathcal{X} \subseteq \mathbb{P}^{k}(K)$ consisting of $n=: \operatorname{deg} \mathcal{X} \geq 1$ pairwise different $K$-rational points. By

$$
I_{\mathcal{X}}:=(\{F \in R \text { homogenous } \mid F(P)=0 \text { for all } P \in \mathcal{X}\}),
$$

we denote its homogenous vanishing ideal. Let $S:=\bigoplus_{d \geq 0} S_{d}:=R / I_{\mathcal{X}}$ and

$$
H_{\mathcal{X}}(d):=\operatorname{dim}_{K}\left(S_{d}\right)
$$

(for $d \in \mathbb{N}$ ) the Hilbert function of $\mathcal{X}$. The Castelnuovo-Mumford regularity of $\mathcal{X}$ is the uniquely determined number $r_{\mathcal{X}}$ such that

$$
H_{\mathcal{X}}(d)=n \text { for } d \geq r_{\mathcal{X}} \text { and } H_{\mathcal{X}}\left(r_{\mathcal{X}}-1\right) \leq n-1 \text {. }
$$

It is well known that $H_{\mathcal{X}}$ is strictly increasing for $0 \leq d \leq r_{\mathcal{X}}$; in particular, $r_{\mathcal{X}} \leq n-1$.

2010 AMS Mathematics subject classification. Primary 14G15.

Received by the editors on September 18, 2013, and in revised form on January 2, 2014. 
From now on, we assume that $K=\mathbb{F}_{q}$ is the finite field with $q$ elements, where $q$ is an arbitrary prime power. One would like to know which Hilbert functions $H_{\mathcal{X}}$ respectively, for which regularities $r_{\mathcal{X}}$ are possible. For infinite fields $K$, the answer to the first (and hence also to the second) question was given by Geramita, Maroscia and Roberts ([6, Sections 1 and 3]).

$r_{\mathcal{X}}$ has the following geometric description:

Remark. $r_{\mathcal{X}}$ is the smallest number such that for every $P \in \mathcal{X}$, there exists a hypersurface $H_{P} \subseteq \mathbb{P}_{\mathbb{F}_{q}}^{k}$ defined over $\mathbb{F}_{q}$, of degree $r_{\mathcal{X}}$ which separates $P$ from $\mathcal{X}$, that is $H_{P} \cap \mathcal{X}=\mathcal{X} \backslash\{P\}$.

Therefore, the following definition of $s(n, q)$ agrees with the one from the abstract:

$$
\begin{aligned}
s(n, q) & =\max \left\{r_{\mathcal{X}} \mid \text { there exist } k \geq 1, \mathcal{X} \subseteq \mathbb{P}^{k}\left(\mathbb{F}_{q}\right) \text { with } \operatorname{deg} \mathcal{X}=n\right\} \\
& =\max \left\{r_{\mathcal{X}} \mid \mathcal{X} \subseteq \mathbb{P}^{n-1}\left(\mathbb{F}_{q}\right), \operatorname{deg} \mathcal{X}=n\right\}
\end{aligned}
$$

(the latter holds since the embedding dimension of $\mathcal{X}$ is at most $n-1$ ).

It is known ([8, Lemma 1.2]) that

$$
s(n, q) \leq s(n+1, q) \leq s(n, q)+1 \text { for } n \in \mathbb{N}_{>0} .
$$

The function $s(n, q)$ can be extended to a step function $s(x, q)$ on $\mathbb{R}_{>0}$, its steps ("jump discontinuities") have height 1 and are precisely at those $x=n \in \mathbb{N}_{>1}$ where $s(n, q)=s(n-1, q)+1$. Trivially, the function $s(x, q)$ is determined by its initial value $s(1, q)=0$ and its jump discontinuities $a_{1}<a_{2}<\ldots$ For $q=2$ and $q=3$, the function $s(n, q)$ was completely computed in ([8, Corollary 1.4]). So far, for $q \geq 4$, the following was known (loc. cit.):

a) $a_{i}=i+1$ for $i=1, \ldots, q-1$.

b) $a_{(m-1)(q-1)+1}=\left(q^{m}-1\right) /(q-1)$ and $a_{m(q-1)}=q^{m}$ for every $m \geq 2$.

c) For every $m \geq 2$ and for $r=2, \ldots, q-2$, the jump discontinuity $a_{(m-1)(q-1)+r}$ is in the half-open interval $I_{m, r}=\left(r\left(q^{m}-1\right) /(q-1),(r+\right.$ 1) $\left.q^{m-1}\right]$, but its precise position was unknown. For $m=2$, we show

Proposition 1.1. For $q \geq 4$ and $r=2, \ldots, q-2$,

$$
a_{q-1+r}=(r+1) q
$$


i.e., the first $2 q-1$ jump discontinuities are: $2, \ldots, q, q+1,3 q, \ldots,(q-$ 1) $q, q^{2}, q^{2}+q+1$. Therefore, $s(x, q)$ is known in the interval $\left[1,2\left(q^{2}+\right.\right.$ $q+1)]$.

One may conjecture that the unknown jump discontinuities of $s(x, q)$ are at the right edges of the intervals $I_{m, r}$.

In the proof of this proposition we will study, for $1 \leq k<n \leq$ $\left(q^{k+1}-1\right) /(q-1)$ (i.e., where it makes sense), the invariants

$$
s(n, k, q):=\max \left\{r_{\mathcal{X}} \mid \mathcal{X} \subseteq \mathbb{P}^{k}\left(\mathbb{F}_{q}\right) \text { nondegenerate and of degree } n\right\}
$$

(recall that a set $\mathcal{X} \subseteq \mathbb{P}_{\mathbb{F}_{q}}^{k}$ is nondegenerate if it spans the whole space). [7, Cor. 2.2 a)] says that $s(n, k, q)$ is increasing in $n$. In contrast to this:

Proposition 1.2. $s(n, k, q)$ is decreasing in $k$.

Together with [7, Proposition 1.6] we shall see that this already implies Proposition 1.1. In addition, we are able to show the following improvement of [7, Proposition 1.4b)]:

Proposition 1.3. For every $k \geq 2$ (and every prime power $q$ ),

$$
s(2 q+k, k, q)=q
$$

(note that the left hand side is well-defined since $k<2 q+k \leq$ $\left.q^{k}+q^{k-1}+\cdots+1\right)$.

We shall now define and study the following affine version of the function $s(n, q)$ : Embed $\mathbb{A}^{k}\left(\mathbb{F}_{q}\right)$ into $\mathbb{P}^{k}\left(\mathbb{F}_{q}\right)=\left\{\mathbb{F}_{q} \cdot v \mid v \in \mathbb{F}_{q}^{k+1} \backslash\{0\}\right\}$ by $\left(x_{1}, \ldots, x_{k}\right) \mapsto\left\langle 1, x_{1}, \ldots, x_{k}\right\rangle=\mathbb{F}_{q} \cdot\left(1, x_{1}, \ldots, x_{k}\right)$. For an arbitrary set $\mathcal{X} \subseteq \mathbb{A}^{k}\left(\mathbb{F}_{q}\right)$, by a remark from above, $r_{\mathcal{X}}$ is the smallest number $r$ such that any interpolation problem

$$
\varphi(P)=w_{P}\left(\text { for } P \in \mathcal{X}, w_{P} \in \mathbb{F}_{q}\right)
$$

has a polynomial solution $\varphi$ of degree $\leq r\left(r_{\mathcal{X}}\right.$ is the interpolation degree of $\mathcal{X}$ in the sense of [3, section $4 \mathrm{~A}])$. 
Definition 1.4. a) We call a subset $\mathcal{X} \subseteq \mathbb{P}^{k}\left(\mathbb{F}_{q}\right)$ affine if there exists a hyperplane $H \subseteq \mathbb{P}_{\mathbb{F}_{q}}^{k}$, defined over $\mathbb{F}_{q}$ and disjoint from $\mathcal{X}$.

b) $s_{a}(n, q):=\max \left\{r_{\mathcal{X}} \mid\right.$ there exist $k \geq 1, \mathcal{X} \subseteq \mathbb{P}^{k}\left(\mathbb{F}_{q}\right), \mathcal{X}$ affine, $\operatorname{deg} \mathcal{X}=n\}$.

By what was just said, this definition agrees with the one from the abstract. The following proposition describes $s_{a}(n, q)$ completely:

Proposition 1.5. Let $r, m, n \in \mathbb{N}_{>0}$ and $r \leq q-1$.

For $r q^{m-1} \leq n<(r+1) q^{m-1}$,

$$
s_{a}(n, q)=(m-1)(q-1)+r-1 .
$$

It turns out (see Section 4) that this is a simple application of the Cayley-Bacharach conjecture ([4, CB12]). However, with regard to the function $s$ of our main interest, we have:

Remark 1.6. The functions $s_{a}$ and $s$ are different.

In fact, for any $m \geq 2$, by [8, Theorem 1.3$]$,

$$
s\left(\frac{q^{m}-1}{q-1}, q\right)=(m-1)(q-1)+1,
$$

whereas, by Proposition 1.5 with $r=1$

$$
s_{a}\left(\frac{q^{m}-1}{q-1}, q\right)=(m-1)(q-1) .
$$

2. The function $s(n, k, q)$ and proofs of $1.1,1.2$. The invariants $s(n, k, q)$ are finer than $s(n, q)$ : It is easily seen that one always has

$$
s(n, q)=\max \left\{s(n, k, q) \mid 1 \leq k<n \leq \frac{q^{k+1}-1}{q-1}\right\} .
$$

$s(n, k, q)$ was studied by Kreuzer and the second author in [7]:

$s(n, k, q)$ is increasing in $n([7$, Corollary 2.2a) $])$ and $s(n, k, q)$ was completely computed in both cases $q=2$ and $k=2$ ([7, Proposition 1.2, respectively, Proposition 1.6]). 
Proof that $s(n, k, q)$ is decreasing in $k$ (Proposition 1.2). Let $q=p^{e}$ be a prime power, $e \geq 1$ and

$$
2 \leq k<n \leq \frac{q^{k}-1}{q-1}\left(=\left|\mathbb{P}^{k-1}\left(\mathbb{F}_{q}\right)\right|\right) .
$$

We have to show that $s(n, k, q) \leq s(n, k-1, q)$. It is clear from our hypothesis that both numbers $s(n, k, q)$ and $s(n, k-1, q)$ are defined. Now, let $\mathcal{X}=\left\{P_{1}, \ldots P_{n}\right\} \subseteq \mathbb{P}^{k}\left(\mathbb{F}_{q}\right)$ be nondegenerate of degree $n$ and $r_{\mathcal{X}}=s(n, k, q)$.

In any case the dimension of the $\mathbb{F}_{q}$-vector space

$$
\left(\mathbb{F}_{q}\left[X_{0}, \ldots, X_{k}\right] / I_{\mathcal{X}}\right)_{r_{\mathcal{X}}-1}
$$

is smaller than $n$; therefore, according to the remark from the introduction, $\mathbb{F}_{q}[\underline{X}]:=\mathbb{F}_{q}\left[X_{0}, \ldots, X_{k}\right]$ contains no homogenous polynomial $p$ of degree $r_{\mathcal{X}}-1$ with (if necessary we renumber the points $P_{i}$ )

$$
\begin{array}{r}
P_{1} \notin V^{+}(p) \\
P_{2}, \ldots, P_{n} \in V^{+}(p)
\end{array}
$$

where $V^{+}(p)$ denotes the zero set of $p$ in $\mathbb{P}^{k}\left(\mathbb{F}_{q}\right)$.

Claim. There exists a line $l \subseteq \mathbb{P}^{k}\left(\mathbb{F}_{q}\right)$ with $l \cap \mathcal{X}=\left\{P_{1}\right\}$.

Proof of claim. For the lines $P_{1} \vee P_{i}$ connecting $P_{1}$ with $P_{i}$ we have:

$$
\begin{aligned}
\left|\left(\bigcup_{i=2}^{n} P_{1} \vee P_{i}\right)\right| & \leq 1+(n-1) \cdot q \leq 1+\left(\frac{q^{k}-1}{q-1}-1\right) \cdot q \\
& =\frac{q^{k+1}-q^{2}+q-1}{q-1}<\frac{q^{k+1}-1}{q-1}=\left|\mathbb{P}^{k}\left(\mathbb{F}_{q}\right)\right| .
\end{aligned}
$$

So there is at least one point $P \in \mathbb{P}^{k}\left(\mathbb{F}_{q}\right)$ not on the union of the lines $P_{1} \vee P_{i}$; take $l$ to be the line connecting $P$ and $P_{1}$.

We choose $P \in l \backslash\left\{P_{1}\right\}$ and take the projection with center $P$ :

$$
\mathbb{P}^{k}\left(\mathbb{F}_{q}\right) \backslash\{P\} \stackrel{\pi}{\longrightarrow} \mathbb{P}^{k-1}\left(\mathbb{F}_{q}\right) .
$$

$l=P_{1} \vee P$ connects $P_{1}$ with $P$, and $l \backslash\{P\}$ is the fibre over $\pi\left(P_{1}\right)$. Because of $l \cap \mathcal{X}=\left\{P_{1}\right\}$, the restriction

$$
\pi \mid \mathcal{X}: \mathcal{X} \longrightarrow \mathbb{P}^{k-1}\left(\mathbb{F}_{q}\right)
$$


has only $P_{1}$ in its fibre over $\pi\left(P_{1}\right)$.

Let $Y_{0}, \ldots, Y_{k-1}$ be the coordinates of $\mathbb{P}^{k-1}\left(\mathbb{F}_{q}\right)$. Algebraically, $\pi$ corresponds to a homogenous, injective ring homomorphism

$$
\iota: \mathbb{F}_{q}[\underline{Y}]:=F_{q}\left[Y_{0}, \ldots, Y_{k-1}\right] \longrightarrow \mathbb{F}_{q}\left[X_{0}, \ldots, X_{k}\right]
$$

(under which the $Y_{i}$ are mapped to certain linear forms). The ring $\mathbb{F}_{q}[\underline{Y}]$ contains no polynomial $p_{0}$ of degree $r_{\mathcal{X}}-1$ with

$$
\begin{aligned}
\pi\left(P_{1}\right) & \notin V^{+}\left(p_{0}\right) \\
\pi\left(P_{2}\right), \ldots, \pi\left(P_{n}\right) & \in V^{+}\left(p_{0}\right),
\end{aligned}
$$

because otherwise $\iota\left(p_{0}\right) \in \mathbb{F}_{q}[\underline{X}]$ would be a polynomial of degree $r_{\mathcal{X}}-1$ with $P_{1} \notin V^{+}\left(\iota\left(p_{0}\right)\right), P_{2}, \ldots, P_{n} \in V^{+}\left(\iota\left(p_{0}\right)\right)$.

By construction, $\pi\left(P_{1}\right)$ is not contained in $\left\{\pi\left(P_{2}\right), \ldots, \pi\left(P_{n}\right)\right\}$. In particular, from (2.1) above we conclude

$$
r_{\pi(\mathcal{X})} \geq r_{\mathcal{X}}
$$

and furthermore (note that $\pi(\mathcal{X}) \subseteq \mathbb{P}^{k-1}\left(\mathbb{F}_{q}\right)$ is nondegenerate because $I_{\mathcal{X}}$ contains no linear form, a fortiori $I_{\pi(\mathcal{X})}=I_{\mathcal{X}} \cap \mathbb{F}_{q}[\underline{Y}]$ contains no linear form) by [7, Corollary 2.2a)],

$$
s(n, k-1, q) \geq s(|\pi(\mathcal{X})|, k-1, q) \geq r_{\pi(\mathcal{X})} \geq r_{\mathcal{X}}=s(n, k, q) . \quad \square_{1.2}
$$

Proposition 1.2 implies Proposition 1.1. Note that the first jump discontinuities $a_{1}=2, \ldots, a_{q}=q+1$ as well as $a_{2 q-2}=q^{2}, a_{2 q-1}=$ $q^{2}+q+1$ are known by [8, Corollay 1.4]. To determine the jump discontinuities $a_{q+1}, \ldots, a_{2 q-3}$ which are missing in between (at least for $q \geq 4$ ), we use the following consequence of proposition 1.2:

Corollary 2.1. In the interval $\left(\left(q^{m}-1\right) /(q-1),\left(q^{m+1}-1\right) /(q-1)\right]$, $m \geq 1$, one has

$$
s(n, q)=s(n, m, q) .
$$

Proof. $s(n, k, q)$ is decreasing in $k$ and we simply take the smallest possible value for $k$ where $s(n, k, q)$ is defined.

In particular, for $n \in\left\{q+2, \ldots,\left(q^{3}-1\right) /(q-1)=q^{2}+q+1\right\}$,

$$
s(n, q)=s(n, 2, q)
$$


and the latter function was concretely computed in [7, Proposition 1.6]. Furthermore, by [8, Theorem 1.3a)], $s(n, q)=2 q-1$ for $q^{2}+q+1 \leq$ $n \leq 2\left(q^{2}+q+1\right)$.

Remark 2.2. Let $s_{a}(n, k, q)$ be the largest interpolation degree that any nondegenerate $\mathcal{X} \subseteq \mathbb{A}^{k}\left(\mathbb{F}_{q}\right)$ of degree $n$ can achieve. Similar arguments as above show, that

$$
s_{a}(n, k, q)=s_{a}(n, q), \quad \text { for } q^{k-1}<n \leq q^{k},
$$

hence, by Proposition $1.5, s_{a}(n, k, q)$ is well known in this range.

3. Proof of 1.3. Note that, for every $k \geq 2$ and every prime power $q, s(2 q+k, k, q)$ is defined since $2 q+k \leq q^{k}+\ldots+q+1$. To prove Proposition 1.3, we need some preparations:

Let $K$ be a field and $k \geq 2$. For a vector $a=\left(a_{0}, \ldots, a_{k}\right) \in K^{k+1}$, we call

$$
\operatorname{supp} a:=\left\{i \mid a_{i} \neq 0\right\} \subseteq\{0, \ldots, k\}
$$

its support and

$$
\|a\|:=|\operatorname{supp} a|
$$

its weight. We start with the map

$$
\widetilde{\varphi}: K^{k+1} \rightarrow K^{\left(\begin{array}{c}
k+1 \\
2
\end{array}\right)},\left(a_{0}, \ldots, a_{k}\right) \longmapsto\left(a_{0} a_{1}, \ldots, a_{k-1} a_{k}\right)
$$

(strictly speaking we once and for all fix an arbitrary order on the set of all pairs $\left(a_{i} a_{j}\right)$ for $j>i$ on the right-hand side).

Lemma 3.1. Let $v_{1}, v_{2}, v_{3} \in K^{k+1} \backslash\{0\}$, and write $v_{i}=\left(v_{i j}\right)_{\substack{j=0, \ldots, k \\ i=1,2,3}}$.

(i) Assume that $v_{1}$ and $v_{2}$ have the same support and weight at least three. If $v_{1}$ and $v_{2}$ are linearly independent, then $\widetilde{\varphi}\left(v_{1}\right)$ and $\widetilde{\varphi}\left(v_{2}\right)$ are likewise linearly independent.

(ii) If $v_{1}, v_{2}$ and $v_{3}$ have pairwise different support and $\left\|v_{i}\right\| \geq 2$ for $i=1,2,3$, then $\widetilde{\varphi}\left(v_{1}\right), \widetilde{\varphi}\left(v_{2}\right)$ and $\widetilde{\varphi}\left(v_{3}\right)$ are linearly independent.

Proof. (i) Without loss of generality, we assume that $\{0,1,2\} \subseteq \operatorname{supp} v_{1}$ $\left(=\operatorname{supp} v_{2}\right)$ and that $\operatorname{det}\left(\begin{array}{ll}v_{11} & v_{12} \\ v_{21} & v_{22}\end{array}\right) \neq 0$. Then 


$$
\widetilde{\varphi}\left(v_{i}\right)=\left(v_{i 0} v_{i 1}, v_{i 0} v_{i 2}, \ldots\right), \quad i=1,2,
$$

with

$$
\operatorname{det}\left(\begin{array}{ll}
v_{10} v_{11} & v_{10} v_{12} \\
v_{20} v_{21} & v_{20} v_{22}
\end{array}\right)=v_{10} v_{20} \cdot \operatorname{det}\left(\begin{array}{ll}
v_{11} & v_{12} \\
v_{21} & v_{22}
\end{array}\right) \neq 0 ;
$$

in particular $\widetilde{\varphi}\left(v_{1}\right)$ and $\widetilde{\varphi}\left(v_{2}\right)$ are linearly independent.

(ii) Without loss of generality, $\left\|v_{3}\right\| \leq\left\|v_{2}\right\| \leq\left\|v_{1}\right\|$. The $\left(\begin{array}{c}k+1 \\ 2\end{array}\right)$-tuples $\widetilde{\varphi}\left(v_{1}\right), \widetilde{\varphi}\left(v_{2}\right), \widetilde{\varphi}\left(v_{3}\right)$ have pairwise different support (since this property holds for $\left.v_{1}, v_{2}, v_{3}\right)$. In particular, whenever $i \neq j$, the vectors $\widetilde{\varphi}\left(v_{i}\right)$ and $\widetilde{\varphi}\left(v_{j}\right)$ are linearly independent. We assume to the contrary that $\widetilde{\varphi}\left(v_{1}\right), \widetilde{\varphi}\left(v_{2}\right), \widetilde{\varphi}\left(v_{3}\right)$ are linearly dependent. Since any two of them are linearly independent there exist $\lambda, \mu \in K \backslash\{0\}$ such that

$$
\widetilde{\varphi}\left(v_{3}\right)=\lambda \widetilde{\varphi}\left(v_{1}\right)+\mu \widetilde{\varphi}\left(v_{2}\right) .
$$

$\left\|v_{2}\right\| \leq\left\|v_{1}\right\|$ and $\operatorname{supp} v_{1} \neq \operatorname{supp} v_{2}$; hence, $\operatorname{supp} v_{1} \nsubseteq \operatorname{supp} v_{2}$. Therefore, we may assume that $\operatorname{supp} v_{1}=\{0, \ldots, d\}$ with $1 \leq d \leq k$ and $0 \notin \operatorname{supp} v_{2}$.

$$
\begin{aligned}
& v_{10} v_{11} \neq 0, \ldots, v_{10} v_{1 d} \neq 0 \\
& v_{20} v_{21}=\cdots=v_{20} v_{2 d}=0
\end{aligned}
$$

and $(*)$ implies

$$
v_{30} v_{31}=\lambda v_{10} v_{11} \neq 0, \ldots, v_{30} v_{3 d}=\lambda v_{10} v_{1 d} \neq 0 ;
$$

hence, $\operatorname{supp} v_{1}=\{0, \ldots, d\} \subseteq \operatorname{supp} v_{3}$. Because of $\left\|v_{3}\right\| \leq\left\|v_{1}\right\|$, we get $\operatorname{supp} v_{1}=\operatorname{supp} v_{3}$ which contradicts our hypothesis.

For any given subset $M \subseteq\{0, \ldots, k\},|M| \geq 2$, set

$$
\mathbb{P}_{M}^{k}=\left\{\langle v\rangle \in \mathbb{P}^{k}(K) \mid \operatorname{supp} v=M\right\}
$$

and

$$
\bar{M}:=\operatorname{supp} \widetilde{\varphi}(v), \text { if } \operatorname{supp} v=M
$$

( $\bar{M}$ does not depend on the choice of $v$ ). The map

$$
\widetilde{\varphi}: \mathbb{P}_{M}^{k} \longrightarrow \mathbb{P}_{\bar{M}}^{\left(\begin{array}{c}
k+1 \\
M
\end{array}\right)-1},\langle v\rangle \longmapsto\langle\widetilde{\varphi}(v)\rangle
$$

is well defined and Lemma 3.1.i. implies: 


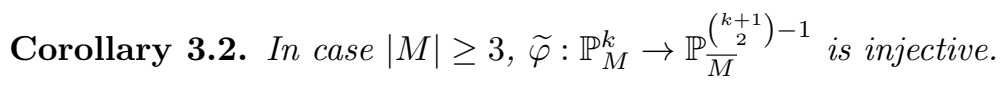

Furthermore we need [8, Remark 5.1] in the following form: Let $\mathcal{X}=\left\{P_{1}, \ldots, P_{n}\right\} \subseteq \mathbb{P}^{k}\left(\mathbb{F}_{q}\right), \operatorname{deg} \mathcal{X}=n$. For every $i$, choose $v_{i} \in \mathbb{F}_{q}^{k+1}$ with $P_{i}=\left\langle v_{i}\right\rangle$. Define

$$
e v_{d}: R_{d} \rightarrow \mathbb{F}_{q}^{n}, F \longmapsto\left(F\left(v_{1}\right), \ldots, F\left(v_{n}\right)\right)^{T} ; \quad V^{(d)}:=\operatorname{im}\left(e v_{d}\right)
$$

Then $\operatorname{ker}\left(e v_{d}\right)=\left(I_{\mathcal{X}}\right)_{d}$, and hence

$$
\operatorname{dim} V^{(d)}=\operatorname{dim} R_{d} /\left(I_{\mathcal{X}}\right)_{d} .
$$

By $A_{d}$, we denote the coefficient matrix of $e v_{d}$ with respect to the basis $\mathcal{B}=\left\{X^{\alpha}|| \alpha \mid=d\right\}$ of $R_{d}$. We have $H_{\mathcal{X}}(d)=\operatorname{rank} A_{d}$. The rows of $A_{d}$ are the vectors $\left(X^{\alpha}\left(v_{i}\right)\left|\alpha \in \mathbb{N}^{k+1},\right| \alpha \mid=d\right.$ ), for $i=1, \ldots, n$ (assuming $\mathcal{B}$ is suitably ordered).

Proof of Proposition 1.3. By [7, Proposition 1.4b)] one has $s(2 q+k-$ $1, k, q)=q$ and, by using [7, Proposition 2.1e)] twice, it is easy to see that

$$
q \leq s(2 q+k, k, q) \leq q+1 .
$$

Therefore, we have to show $r_{\mathcal{X}} \neq q+1$ for every $\mathcal{X} \subseteq \mathbb{P}^{k}\left(\mathbb{F}_{q}\right)$, nondegenerate and with $\operatorname{deg} \mathcal{X}=2 q+k$.

Claim. $H_{\mathcal{X}}(2) \geq k+4$.

Proof of claim. Without loss of generality, we may assume that $\mathcal{X}_{1}:=\left\{\left\langle e_{0}\right\rangle, \ldots,\left\langle e_{k}\right\rangle\right\} \subseteq \mathcal{X}$, where $e_{i}$ is the $i$ th standard basis vector in $\mathbb{F}_{q}^{k+1}$. Let $v_{1}, \ldots, v_{2 q-1} \in \mathbb{F}_{q}^{k+1}$ be such that

$$
\mathcal{X}=\mathcal{X}_{1} \cup\left\{\left\langle v_{1}\right\rangle, \ldots,\left\langle v_{2 q-1}\right\rangle\right\} .
$$

We define

$$
\begin{aligned}
\varphi: \mathbb{F}_{q}^{k+1} & \longrightarrow \mathbb{F}_{q}^{\left(\begin{array}{c}
k+2 \\
2
\end{array}\right)} \\
a=\left(a_{0}, \ldots, a_{k}\right) & \longmapsto\left(a_{0}^{2}, \ldots, a_{k}^{2}, a_{0} a_{1}, \ldots, a_{k-1} a_{k}\right) \\
& =\left(X^{\alpha}(a)|| \alpha \mid=2\right) .
\end{aligned}
$$


The rows of $A_{2}$ are $\varphi\left(e_{0}\right), \ldots, \varphi\left(e_{k}\right), \varphi\left(v_{1}\right), \ldots, \varphi\left(v_{2 q-1}\right)$ :

$$
A_{2}=\left(\begin{array}{ccc|c}
1 & & 0 & \\
& \ddots & & 0 \\
0 & & 1 & \\
\hline & * & & \widetilde{A}
\end{array}\right), \quad \text { where } \widetilde{A}=\left(\begin{array}{c}
\widetilde{\varphi}\left(v_{1}\right) \\
\vdots \\
\widetilde{\varphi}\left(v_{2 q-1}\right)
\end{array}\right) \text {, }
$$

with $\widetilde{\varphi}$ being taken from Lemma 3.1. To prove our claim $H_{\mathcal{X}}(2) \geq k+4$, we have to show that $\operatorname{rank} \widetilde{\mathrm{A}} \geq 3$ (since $H_{\mathcal{X}}(2)=\operatorname{rankA}_{2}=\mathrm{k}+1+$ $\operatorname{rank} \widetilde{\mathrm{A}})$ :

Let $M \subseteq\{0, \ldots, k\},|M| \geq 2$ and $\mathcal{X}_{M}:=\mathcal{X} \cap \mathbb{P}_{M}^{k}\left(=\left(\mathcal{X} \backslash \mathcal{X}_{1}\right) \cap \mathbb{P}_{M}^{k}\right)$. Clearly, since every line has exactly $q+1 \mathbb{F}_{q}$-rational points,

$$
\begin{gathered}
\left|L \cap \mathbb{P}_{M}^{k}\right| \leq q-1 \text { for every line } L \subseteq \mathbb{P}^{k}\left(\mathbb{F}_{q}\right) \\
\text { If }|M|=2, \text { then }\left|\mathbb{P}_{M}^{k}\right|=q-1 .
\end{gathered}
$$

To finish the proof of our claim, we distinguish between two cases:

(a) If $\mathcal{X} \backslash \mathcal{X}_{1}$ contains three points $\left\langle w_{1}\right\rangle,\left\langle w_{2}\right\rangle$ and $\left\langle w_{3}\right\rangle$ with pairwise different supports, then the vectors $\widetilde{\varphi}\left(w_{1}\right), \widetilde{\varphi}\left(w_{2}\right)$ and $\widetilde{\varphi}\left(w_{3}\right)$ are linearly independent and $\operatorname{rank} \widetilde{\mathrm{A}} \geq 3$, by Lemma 3.1.ii.

(b) If there are at most two $M$ with $|M| \geq 2$ and $\mathcal{X}_{M} \neq \emptyset$, then, because of $\left|\mathcal{X} \backslash \mathcal{X}_{1}\right|=2 q-1=q+q-1$, there exists such an $M$ with $\left|\mathcal{X}_{M}\right| \geq q$. By equation (3.2), we get $|M| \geq 3$ and then, by Corollary 3.2, $\left|\widetilde{\varphi}\left(\mathcal{X}_{M}\right)\right| \geq q$. By equation (3.1) it is clear that the set

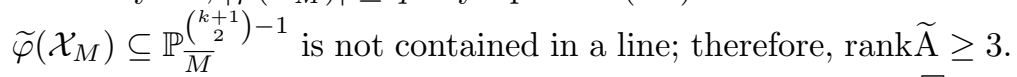

$H_{\mathcal{X}}(2)=k+1+\operatorname{rank} \widetilde{\mathrm{A}} \geq \mathrm{k}+4$. Assume that $r_{\mathcal{X}}=q+1$ : The first difference function $\Delta H_{\mathcal{X}}=H_{\mathcal{X}}(d)-H_{\mathcal{X}}(d-1)$ has the form

$$
\Delta H_{\mathcal{X}}: 1, k, h_{2}, h_{3}, \ldots, h_{q+1}, 0,0, \ldots \text { with } h_{j} \geq 1 \quad(j=2, \ldots, q+1) .
$$

$H_{\mathcal{X}}(2) \geq k+4$ implies

$$
h_{2}=H_{\mathcal{X}}(2)-H_{\mathcal{X}}(1) \geq k+4-(k+1)=3 .
$$

Furthermore, we have $h_{j} \geq 2$ for $j=3, \ldots, q$. If $h_{j}$ was equal to 1 for some $j \in\{3, \ldots, q\}$, then, by [7, Proposition $2.1 \mathrm{c})$ ], also both $h_{q}$ and $h_{q+1}$ would be equal to 1 ; by [7, Proposition $2.1 \mathrm{~d}$ )], there would be a 
line $L \subseteq \mathbb{P}^{k}\left(\mathbb{F}_{q}\right)$ with

$$
|\mathcal{X} \cap L| \geq r_{\mathcal{X}}+1=q+2>q+1=|L|,
$$

(in this context, see also [6, Proposition 5.2]), which is absurd.

Hence, we finally get

$$
\begin{aligned}
\operatorname{deg} \mathcal{X} & =\sum_{d \in \mathbb{N}} \Delta H_{\mathcal{X}}(d) \\
& =1+k+h_{2}+\left(h_{3}+\ldots+h_{q}\right)+h_{q+1} \\
& \geq 1+k+3+(q-2) \cdot 2+1 \\
& =2 q+k+1
\end{aligned}
$$

which contradicts our assumptions. Therefore, $r_{\mathcal{X}} \neq q+1$.

4. Proof of Proposition 1.5. Similarly to [8, Lemma 1.2], we have

Remark 4.1. For all $n \in \mathbb{N}_{>0}$,

$$
s_{a}(n, q) \leq s_{a}(n+1, q) \leq s_{a}(n, q)+1 .
$$

Proof of Proposition 1.5. From the proof of [8, Proposition $1.6 \mathrm{~b})$ ], we know that there is an affine complete intersection $\mathcal{X} \subseteq \mathbb{A}^{m}\left(\mathbb{F}_{q}\right) \subseteq$ $\mathbb{P}^{m}\left(\mathbb{F}_{q}\right)$ of degree $r q^{m-1}$ and regularity $(m-1)(q-1)+r-1$; hence,

$$
\begin{aligned}
s_{a}(n, q) & \geq s_{a}\left(r q^{m-1}, q\right) \geq r_{\mathcal{X}} \\
& =(m-1)(q-1)+r-1 \quad \text { for } n \geq r q^{m-1} .
\end{aligned}
$$

Conversely, let $k \geq 1$ and $\mathcal{X} \subseteq \mathbb{P}^{k}\left(\mathbb{F}_{q}\right)$ be affine with $\operatorname{deg} \mathcal{X}<$ $(r+1) q^{m-1}$. We have to show that $r_{\mathcal{X}} \leq(m-1)(q-1)+r-1$ and may assume that $\mathcal{X}$ does not meet the hyperplane $X_{0}=0$. Then, for $\bar{S}:=R / I_{\mathcal{X}}+\left(X_{0}\right)=\mathbb{F}_{q}\left[X_{1}, \ldots, X_{k}\right] / J$,

$$
\left\{X_{1}^{q}, \ldots, X_{k}^{q}\right\} \subseteq J \text { and } \operatorname{dim}_{\mathbb{F}_{q}} \bar{S}=\operatorname{deg} \mathcal{X}<(r+1) q^{m-1} .
$$

Finally, by the following simple combinatorial lemma, we have $\bar{S}_{d}=0$ for $d=(m-1)(q-1)+r$, i.e., $r_{\mathcal{X}} \leq(m-1)(q-1)+r-1$.

Lemma 4.2. Let $k, m$ and $q$ be natural numbers, $k \geq 1$ and $1 \leq r \leq$ $q-1$. Let $\alpha:=\left(\alpha_{1}, \ldots, \alpha_{k}\right) \in \mathbb{N}^{k}$ be of degree $|\alpha|:=\alpha_{1}+\ldots+\alpha_{k}=$ 


$$
\begin{gathered}
m(q-1)+r \text { and such that } 0 \leq \alpha_{j} \leq q-1 \text { for } j=1, \ldots, k \text {. Then } \\
\left(\alpha_{1}+1\right) \cdot \ldots \cdot\left(\alpha_{k}+1\right) \geq(r+1) q^{m} .
\end{gathered}
$$

This follows from [8, Lemma $2.2 \mathrm{~b}$ )] and is easily seen anyway.

Assume $\bar{S}_{d} \neq 0$ for $d=(m-1)(q-1)+r$. By Macaulay's theorem [1, Theorem 4.2.3], there is an order ideal $\mathfrak{M}$ of monomials in $\mathbb{F}_{q}\left[X_{1}, \ldots, X_{k}\right]$ such that the elements $X^{\alpha}+J, X^{\alpha} \in \mathfrak{M}$ form an $\mathbb{F}_{q}$-basis of $\bar{S}$. Since $\bar{S}_{d} \neq 0$ and $\left\{X_{1}^{q}, \ldots, X_{k}^{q}\right\} \subseteq J$, there is a monomial $X^{\alpha} \in \mathfrak{M}\left(0 \leq \alpha_{j} \leq q-1\right.$ for $\left.j=1, \ldots, k\right)$ of degree $d$. Hence, by Lemma $4.2, \operatorname{dim}_{\mathbb{F}_{q}} \bar{S}=|\mathfrak{M}| \geq \mid\left\{X^{\beta} \mid X^{\beta}\right.$ divides $\left.X^{\alpha}\right\} \mid=$ $\left(\alpha_{1}+1\right) \cdot \ldots \cdot\left(\alpha_{k}+1\right) \geq(r+1) q^{m-1}$, a contradiction.

Alternatively, $\bar{S}_{d}=0$ by the AU-conjecture [5, Conjecture 3.5], which is known to be true for pure powers (see [2]).

5. More general considerations. For $k \geq 1, q \geq 2$ (not necessarily a prime power $)$, let $I(k, q) \subseteq \mathbb{Z}\left[X_{0}, \ldots, X_{k}\right]$ be the ideal generated by the $2 \times 2$-minors of the matrix $\left(\begin{array}{ccc}X_{0}^{q} & \ldots & X_{k}^{q} \\ X_{0} & \ldots & X_{k}\end{array}\right)$.

For instance, if $q$ is a prime power, then $I(k, q) \cdot \mathbb{F}_{q}\left[X_{0}, \ldots X_{k}\right]$ is the homogenous vanishing ideal of $\mathcal{X}=\mathbb{P}^{k}\left(\mathbb{F}_{q}\right) \subseteq \mathbb{P}_{\mathbb{F}_{q}^{k}}^{k}$. More generally, let $K$ be the cyclotomic extension of degree $q-1$ of $\mathbb{Q}$ or of a prime field $\mathbb{F}_{l}$ with $l \nmid(q-1)$. Then $I(k, q)$ defines a smooth finite subscheme $\mathcal{P}_{q}^{k}(K) \subseteq \mathbb{P}^{k}(K) \subseteq \mathbb{P}_{K}^{k}$ of degree $\left(q^{k+1}-1\right) /(q-1)$ and its ideal is given by $I(k, q) \cdot R$ (note that this ideal is saturated).

Questions. What are the Hilbert functions of the subschemes $\mathcal{X} \subseteq \mathcal{P}_{q}^{k}(K)$ ? Does the answer depend on $K$ ? A simpler problem is: which numbers occur as the regularities of such $\mathcal{X}$ of a given degree $n$ ? Find a formula for

$s(n, q ; K):=\max \left\{r_{\mathcal{X}} \mid\right.$ there exist $k \geq 1, \mathcal{X} \subseteq \mathcal{P}_{q}^{k}(K)$ with $\left.\operatorname{deg} \mathcal{X}=n\right\}$ And, again, does $s(n, q ; K)$ depend on $K$ ?

These considerations were suggested by the referee of the paper [8] and are motivated by the following results. Analyzing the proof of Theorem 1.3 in [8], we see that its statements remain true if one allows $q$ to be an arbitrary integer $\geq 2$ and replaces $\mathbb{F}_{q}$ by a cyclotomic field 
$K$, as above. In particular, if $q$ is a prime power we have

$$
s(n, q ; K)=s(n, q)
$$

for all such $K$ and all $n$ for which Theorem 1.3 (loc. cit.) applies. Moreover, the functions $s(n, 2 ; K)=s(n, 2)$ and $s(n, 3 ; K)=s(n, 3)$ are well known and independent from $K$.

Acknowledgments. We thank Martin Kreuzer for his valuable comments to the proof of Proposition 1.4.

\section{REFERENCES}

1. W. Bruns and J. Herzog, Cohen-Macaulay rings, Cambr. Stud. Adv. Math. 39, Cambridge University Press, Cambridge, 1993.

2. G.F. Clements and B. Lindström, A generalization of a combinatorial theorem of Macaulay, J. Combin. Theor. 7 (1969), 230-238.

3. D. Eisenbud, The geometry of syzygies, Grad. Texts Math. 229, Springer, New York, 2005.

4. D. Eisenbud, M. Green and J. Harris, Cayley-Bacharach theorems and conjectures, Bull. Amer. Math. Soc. 33 (1996), 295-324.

5. A.V. Geramita and M. Kreuzer, On the uniformity of zero-dimensional complete intersections, J. Algebra 391 (2013), 82-92.

6. A.V. Geramita, P. Maroscia and L.G. Roberts, The Hilbert function of a reduced k-algebra, J. Lond. Math. Soc. 28 (1983), 443-452.

7. M. Kreuzer and R. Waldi, On the Castelnuovo-Mumford regularity of a projective system, Comm. Alg. 25 (1997), 2919-2929.

8. E. Kunz and R. Waldi, On the regularity of configurations of $\mathbb{F}_{q}$-rational points in projective space, J. Comm. Alg. 5 (2013), 269-280.

Fakultät für Mathematik, Universität Regensburg, D 93040 Regensburg, GERMANY

Email address: michael.hellus@mathematik.uni-regensburg.de

Fakultät für Mathematik, Universität Regensburg, D 93040 Regensburg, GERMANY

Email address: rolf.waldi@mathematik.uni-regensburg.de 